\title{
Individuação e Coping em Adolescentes de Famílias Tradicionais e Divorciadas
}

\author{
Catarina Pinheiro Mota ${ }^{1}$ \\ Departamento de Educação e Psicologia da Universidade de Trás-os-Montes e Alto Douro, \\ Vila Real, Portugal \\ Centro de Psicologia da Universidade do Porto, Porto, Portugal
}

\begin{abstract}
Resumo
O divórcio é marcado por mudanças e a necessidade de reorganização familiar. A literatura tem abordado as implicações deste processo no desenvolvimento dos adolescentes, nomeadamente a existência de aspetos como a qualidade da vinculação aos pais que potenciam o desenvolvimento de coping e protegem face a fatores de risco. O presente estudo tem como objetivo analisar o processo de individuação face às figuras parentais, bem como o seu papel no desenvolvimento de estratégias de coping em adolescentes de diferentes configurações familiares (tradicionais e divorciadas). A amostra foi composta por 399 participantes, com idades entre 15 e 18 anos, de ambos os gêneros, e provenientes de famílias tradicionais e divorciadas/separadas. Os resultados obtidos demonstraram associações entre a individuação parental e o coping. Verificou-se ainda que a configuração familiar não apresenta um efeito moderador na associação entre a individuação parental e o coping, sendo que o divórcio parece não se constituir per si um fator que influencia necessariamente de forma negativa o desenvolvimento do coping em adolescentes, destacando-se a importância da individuação parental neste processo.
\end{abstract}

Palavras-chave: Individuação, coping, adolescência, configuração familiar.

\section{Individuation and Coping in Adolescents from Traditional and Divorced Families}

\begin{abstract}
Divorce is marked by change and the need of family reorganization. The literature has addressed the implications of this process in the development of adolescents, namely the existence of aspects like quality of attachment with parents that improve the development of coping and protect from risk factors. This study aims to analyse the process of individuation to parental figures, as well as, its role in the development of coping strategies in adolescents from different family configurations (traditional and divorced). The sample comprised 399 participants aged 15 to 18 from both genders and from intact and divorced/separated families. The results showed associations between parental individuation and coping. It has been found that familiar configuration doesn't have a moderating effect on the association between parental individuation and coping, and that divorce doesn't seem itself a negatively influences the development of coping in adolescents, highlighting the importance of parental individuation in this process.
\end{abstract}

Keywords: Individuation, coping, adolescence, family configuration.

Endereço para correspondência: Universidade de Trás-os-Montes e Alto Douro, Departamento de Educação e Psicologia, Quinta dos Prados, Edifício das Ciências Humanas e Sociais - Polo I, Vila Real, 5000-801. E mail: catppmota@utad.pt

Estudo financiado parcialmente pela Fundação para a Ciência e a Tecnologia (FCT) ao abrigo do projeto PEst-C/PSI/UI0050/2011 e pelo Fundo Europeu de Desenvolvimento Regional (FEDER) com fundos do programa COMPETE ao abrigo do projeto FCOMP-01-0124-FEDER-022714. 


\section{Individuación y Coping en Adolescentes de Familias Tradicionales y Divorciadas}

\section{Resumen}

El divorcio está marcado por el cambio y la necesidad de una reorganización familiar. La literatura ha considerado las consecuencias de este proceso en el desarrollo de los adolescentes, a saber, la existencia de aspectos tales como la calidad del apego a los padres que mejoran o desarrollo del coping y protegen contra los factores de riesgo. Este estudio tiene como objetivo analizar el proceso de individuación en lo que concirne a las figuras parentales, así como su papel en el desarrollo de estrategias de coping en adolescentes de diferentes configuraciones familiares (tradicionales y divorciadas). La muestra consistió en 399 participantes, de edades comprendidas entre 15 y 18 años, de ambos gêneros, y de familias tradicionales y divorciadas/separadas. Los resultados mostraron asociaciones entre la individuación de los padres y el coping. También se observó que la configuración de la familia no tiene un efecto moderador sobre la asociación entre la individuación de los padres y el coping, por lo que el divorcio no parece constituir per se un factor que necesariamente influye negativamente en el desarrollo del coping en adolescentes, destacando la importancia de la individuación de los padres en este proceso.

Palabras clave: Individuación, coping, adolescencia, configuración familiar.

\section{A Individuação e a Configuração Familiar no Desenvolvimento do Coping}

A vinculação constitui uma tendência inata do ser humano para se ligar a figuras significativas de afeto, desenvolvendo laços afetivos fortes que têm continuidade ao longo do ciclo vital (Ainsworth, 1969; Bowlby, 1988). Esta ligação afetiva caracteriza-se pela sua permanência no tempo, facilitando a procura de proximidade, quer física quer emocional, sendo especialmente relevante em situações geradoras de stress. Ainsworth (1969) desenvolveu o conceito de base segura, com o intuito de explicar a importância da qualidade da ligação estabelecida num ambiente de proteção, que garante ao indivíduo uma estabilidade emocional capaz de criar uma imagem positiva de si e do outro, facultando-lhe maior segurança na exploração do mundo que o rodeia. Fleming (2005) sublinha a importância da criança e mais tarde do adolescente de desenvolver o sentimento de segurança e confiança no outro, no sentido de facilitar a relação com o exterior, nomeadamente no desenvolvimento de comportamentos de exploração.

A adolescência constitui uma etapa desenvolvimental pautada pelas mudanças e reorganizações da relação com as figuras parentais, denotando-se uma necessidade de proximidade com o exterior, nomeadamente com os pares e outras figuras significativas como o par amoroso (e.g. Cohen, Kasen, Chen, Hartmark, \& Gordon, 2003; Eccles, Templeton, Barber, \& Stone, 2003; Lansu \& Cillessen, 2012). Nesta medida assiste-se a uma procura de integração e aceitação fora do contexto parental, o que reflete um sentimento de pertencimento e valorização pessoal. Por outro lado, persiste a necessidade de manutenção do laço parental, fonte impreterível de segurança, mesmo que fisicamente mais tênue na relação direta. Assim, a qualidade da vinculação está subjacente ao processo de individuação, pelo que adolescentes seguros são caracterizados pela capacidade de se autonomizarem, embora avaliando as suas relações com as figuras parentais enquanto bases seguras (Buhl, 2008a; Komidar, Zupančič, Sočan, \& Puklek Levpušček, 2013). A individuação é caracterizada pela independência do jovem à autoridade parental, marcada pela mudança de autoridade unilateral para uma autoridade de cooperação e de reciprocidade, com tendência à progressão durante a adolescência e a jovem adultez (Mota \& Rocha, 2012). Scabini e Manzi (2011) acrescentam que um contexto familiar favorável, com relacionamentos de qualidade, propicia a manutenção dos laços emocionais e um processo de 
individuação bem sucedido. Não se trata de uma tarefa desenvolvimental que se coloca apenas num dado ponto do ciclo vital, mas que se desenrola num contínuo de mudanças sustentadas ao longo das etapas da vida que se vão sucedendo. Assim, percebe-se que as figuras parentais, cada vez mais presentes na vida dos adolescentes, parecem ser um fator facilitador do processo de autonomia. Van Petegem, Beyers, Brenning e Vansteenkiste (2013), num estudo longitudinal realizado com 327 adolescentes, sublinham que uma vinculação evitante e ansiosa aos pais parece assumir relevância na predição da autonomia dos jovens. Os autores apontam que a tomada de decisão pessoal parece estar comprometida recriando jovens mais dependentes e pouco capazes de encontrar estratégias de resolução positivas. Acresce a esta questão que algumas dificuldades podem colocar-se quando existe resistência por parte das figuras parentais no que respeita à separação, permitindo a existência de consequências desadaptativas relativas ao processo de separação-individuação (Mota $\&$ Rocha, 2012). Mayseless e Scharf (2009) sublinham esta ideia, na medida em que ligações desajustadas (pautadas por um elevado controle, triangulação e desvalorização), parecem predizer menores níveis de coping adaptativo, como o evitamento, a fuga e a negação das necessidades de vinculação aos pais. Os adolescentes caracterizados por este tipo de relação com os pais tendem a revelar maiores níveis de ansiedade de controle e conflito no processo de independência emocional (Larkin, Frazer, \& Wheat, 2011). Também Kruse e Walper (2008), observaram num estudo com 649 adolescentes e jovens adultos de diferentes configurações familiares, que jovens de famílias tradicionais com níveis significativos de desajuste relacional na família, sentem-se mais controlados e inibidos, apresentam mais desordens emocionais, mais negação das necessidades e vinculação e tendem a recriar relações inseguras ambivalentes com os demais. Um fenômeno que retrata esta realidade, o estilo parental de envolvimento contínuo e intrusivo, é designado por "pais helicópteros" cuja dinâmica relacional é pautada pela hiperprotecção e o controle, de tal modo que os pais estão incessantemente presentes não permitindo que os adolescentes resolvam as suas próprias questões pessoais (e.g. Coburn, 2006; Rocha \& Rocha, 2012). Esta questão torna-se particularmente relevante quando das transições familiares, nomeadamente em situações de divórcio parental.

O estudo das transições familiares, como o divórcio e a separação dos cônjuges torna-se cada vez mais relevante na compreensão do processo desenvolvimental dos adolescentes (Cano, Gabarra, Moré, \& Crepaldi, 2009). A fase inicial do processo de divórcio é caracterizada por grande sofrimento por parte das crianças e adolescentes, provocando sentimentos de culpa e facilitando o desenvolvimento de processos depressivos, que se poderão manter durante um certo período de tempo após a separação (e.g. Costa \& Mota, 2012). Assim, embora perante um contexto familiar problemático, o processo de divórcio possa ser benéfico para os filhos, grande parte dos adolescentes percebe esta transição familiar como dolorosa (e.g. Amato, 2010; Amato \& Cheadle, 2008; Hack \& Ramires, 2010; Melo \& Mota, 2014). Neste sentido, e partindo das dificuldades que pais e filhos atravessam no decurso das transições familiares, torna-se pertinente perceber o tipo de estratégias de coping que os adolescentes são capazes de desenvolver. O processo de coping refere-se a respostas adaptativas dadas pelo indivíduo perante a avaliação de situações, as quais sente como ameaça, com o intuito de atingir uma adaptação bem sucedida (Folkman \& Moskowitz, 2004). Assim, perante acontecimentos que desencadeiam sentimentos negativos, como poderá ser a dinâmica do processo de divórcio, o indivíduo tende a adotar estratégias que lhe permitam a minimização desse sofrimento (e.g. Amato, 2010; Mathew, Gayman, Turner, Cislo, \& Eliassen, 2011).

Num estudo longitudinal realizado com adolescentes durante 7 anos, Seiffge-Krenke e Beyers (2005), verificaram que as estratégias de coping, bem como o impacto de fatores desenvolvimentais são diferentes para adolescentes com estilos de vinculação segura e insegura. 
Isto porque tanto o estilo de vinculação, como as estratégias de coping desenvolvidas implicam interpretações cognitivas dos eventos geradores de stress. Assim, os adolescentes adotam estratégias de coping mais adaptativas consoante as representações mentais criadas com base nas experiências precoces de vinculação. Os autores sublinham ainda que os adolescentes seguros revelaram um decréscimo do coping retrativo e maior tendência para o uso de coping activo, comparativamente com adolescentes inseguros.

No que diz respeito à capacidade de lidar com eventos geradores de stress, as crianças têm apresentado menos recursos do que adolescentes e adultos, principalmente quando esses eventos implicam as figuras parentais, situações familiares ou domínio escolar (Lisboa et al., 2002). De acordo com Ramires (2004), os adolescentes enfrentam melhor as transições familiares, pois se encontram mais desenvolvidos em termos afetivos, cognitivos e sociais comparativamente com as crianças.

Moura e Matos (2008) num estudo realizado com adolescentes sugerem que devido ao seu desenvolvimento cognitivo, os adolescentes são capazes de perceber melhor as mudanças inerentes ao processo de divórcio, encontrando mais facilmente um suporte alternativo comparativamente com crianças mais novas. Note-se que durante esta descontinuidade familiar, os adolescentes atravessam ainda dificuldades características do período de desenvolvimento da adolescência, o que pode comprometer o processo de separação-individuação. Especialmente quando os pais se mostram menos disponíveis e responsivos, os adolescentes tendem a fechar-se em si ou procurar formas de ajustamento menos adaptativas. Um estudo realizado com 387 adolescentes demonstra a ligação entre o desenvolvimento de vinculação segura e de estratégias de coping adaptativas (Cabral \& Matos, 2007). As autoras apontam que a qualidade do laço emocional estabelecido com as figuras parentais apresentava efeito positivo nas estratégias de coping utilizadas. Por outro lado, constataram que a inibição na exploração e individuação parental se encontrava ligada ao aumento de estratégias de coping desadaptativas, como a retração e a negação das necessidades de vinculação. Assim, o desenvolvimento de estratégias de coping adaptativas por parte dos jovens, durante as transições familiares, poderá relacionar-se com a qualidade das relações com as figuras significativas, uma vez que perante sentimentos de ameaça, a procura de suporte auxilia na forma como gerem as suas angústias (Holmes, 2001). Assim, uma consideração positiva de si, fruto de relações pautadas por proximidade e afeto por parte dos pais, parece potenciar jovens com mais confiança, e por isso mais capazes de se voltarem para o exterior, pedir ajuda de forma ativa e evitar a negação das adversidades (e.g. Assor \& Tal, 2012).

Nesta medida, adolescentes com uma vinculação segura parecem ser mais confiantes, pelo que o seu desenvolvimento sugere a escolha de estratégias de coping mais adaptativas (DiFilippo \& Overholser, 2000). O ajustamento dos adolescentes em situações ansiogénicas e de transição, poderá assim, espelhar-se na forma como gerem as dificuldades (e.g. Mota \& Matos, 2013), muito embora a literatura tenha vindo a suportar a ideia de que outros fatores pessoais, como a personalidade ou o género, possam fazer a diferença no que concerne ao desenvolvimento dos estilos de coping (Seiffge-Krenke, 2011).

\section{Estudo Empírico}

\section{Objetivos}

Este estudo objetiva analisar em que medida o processo de individuação se encontra associado ao desenvolvimento de estratégias de coping em adolescentes de famílias tradicionais e divorciadas. Assim como o contributo da individuação na utilização de diferentes estratégias de coping. Pretende-se ainda compreender o papel moderador da qualidade da configuração familiar na associação entre a individuação e o coping.

\section{Método}

\section{Participantes}

Este estudo foi composto por uma amostra de 389 participantes com idades compreendidas entre os 15 e os 18 anos $(M=16,33 ; D P=1,02)$, 
243 do gênero feminino $(62,5 \%)$ e $146(37,5 \%)$ do gênero masculino. No que se refere ao nível de escolaridade dos participantes, estava compreendida entre o $9^{\circ}$ e o $12^{\circ}$ ano de escolaridade $(M=10,47 ; D P=0,86)$. A idade do pai variou entre os 34 e os 75 anos $(M=47,14 ; D P=5,5)$, enquanto a idade da mãe variou entre os 32 e os 64 anos $(M=44,28 ; D P=4,7)$. No que diz respeito à escolaridade dos pais, em ambas as figuras paterna e materna a média de escolaridade aproximou-se do $9^{\circ}$ ano de escolaridade, $(M=8,56$; $D P=3,83)$ e $(M=9,51 ; D P=3,8)$, respetivamente. A maioria dos participantes vive em famílias tradicionais com 295 adolescentes (75,8\%), 29 (7,5\%) de cônjuges separados e $65(16,7 \%)$ de famílias divorciadas. As últimas duas categorias serão constituídas numa mesma dimensão, uma vez que se assumem famílias onde o adolescente coabita apenas com uma das figuras parentais.

\section{Instrumentos}

O Munich Inventory Test of Adolescence - MITA (Walper, Schwarz, \& Jurasic, 1996; Saraiva, Brandão, \& Matos, 2010) permite a avaliação da individuação bem sucedida em relação à mãe e ao pai, separadamente. É uma escala do tipo Likert de 4 pontos, originalmente com 27 itens. Este instrumento apresenta seis dimensões. No presente estudo foram usadas três dimensões, designadas pelo autor como Relatedness: Manutenção do Laço (3 itens), Negação das Necessidades de Vinculação (5 itens) e Individuação Bem Sucedida (4 itens). Perante a amostra recolhida, foram observados alfas de Cronbach com valores moderadamente altos para ambas as versões (mãe e pai): Manutenção do Laço o valor de alfa de Cronbach é de 0,655 e de 0,795; Negação das Necessidades de Vinculação os valores de alfa são de 0,651 e 0,608; e Individuação Bem Sucedida o valor de alfa é 0,691 e de 0,787 . Estes valores revelam níveis de confiabilidade em cada uma das dimensões permitindo garantir a replicação das medidas dos constructos teóricos. Na execução da análise fatorial confirmatória, na versão mãe, verificam-se índices de ajustamento adequados com $\mathrm{CFI}=0,986, \mathrm{RMESA}=0,042$ e SRMR $=$
0,$045 ; \chi^{2}(12)=20,20 ; p=0,06$. A versão do pai, apresenta igualmente índices de ajustamento adequados: $\mathrm{CFI}=0,983$, RMESA $=0,062 \mathrm{e}$ SRMR $=0,053 ; \chi_{(12)}^{2}=29,78 ; p=0,003$.

O COPE Inventory (Carver, Scheier, \& Kumari Weintraub, 1989; adaptação de Cabral \& Matos, 2004) trata-se de um questionário usado para medir o coping em adolescentes. O COPE é composto por uma escala tipo Likert de 6 pontos. Foi administrada a versão reduzida deste instrumento, constituído por 28 itens e distribuídos por 11 dimensões. Para a análise dos dados foram utilizadas apenas as dimensões Coping Ativo (2 itens), Negação (3 itens) e Uso de Suporte Social e Emocional ( 2 itens). O alfa de Cronbach na amostra recolhida apresenta valores esperados, com uma oscilação de consistência interna semelhante aos valores originais obtidos pelos autores: Coping Ativo $-\alpha=0,583$, Negação $-\alpha$ $=0,630$ e Uso de Suporte Social e Emocional $-\alpha$ $=0,760$. O instrumento apresenta bons índices de ajustamento nas análises fatoriais confirmatórias: $\mathrm{CFI}=0,933, \mathrm{RMESA}=0,072, \mathrm{SRMR}=$ 0,$079 ; \chi_{(37)}^{2}=113,2 ; p=0,001$.

Para a recolha dos dados, foi ainda aplicado um questionário demográfico composto pela identificação do adolescente e identificação escolar, assim como identificação familiar e estado civil dos pais.

\section{Procedimento e Estratégia de Análise de Dados}

Tratando-se de um estudo transversal, os dados foram recolhidos num único momento, recorrendo-se a sete escolas secundárias das regiões norte e centro de Portugal, em ensino regular e profissional. Após a autorização dos respectivos encarregados de educação, e obtenção do termo de consentimento livre e esclarecido, a aplicação do protocolo de investigação foi coletiva, sendo que todos os procedimentos éticos da pesquisa foram salvaguardados. O estudo faz parte de um projeto de investigação aprovado pelo Departamento de Educação e Psicologia da Universidade de Trás-os-Montes e Alto Douro (UTAD), Portugal. As repostas aos questionários ocorreram de uma forma anônima e autônoma, 
ressalvando a confidencialidade e salvaguardando o cariz voluntário da participação. Quando da aplicação dos questionários, explicou-se com clareza os objetivos do estudo, esclarecendo-se dúvidas e outros aspetos pertinentes.

O tratamento dos dados foi realizado com o programa estatístico SPSS - Statistical Package for Social Sciences - na sua versão 20.0 para o sistema Windows. No sentido de identificar e excluir missings e eventuais outliers realizou-se, de forma preliminar, uma limpeza da amostra. Para verificar se os dados da amostra seguiam os pressupostos de normalidade foram analisados os valores de skeweness (assimetria) e kurtosis (achatamento), procedendo-se, concomitantemente, à realização de uma série de análises estatísticas que fornecem informação acerca da distribuição dos dados: teste de Kolmogorov-Smirnov, os gráficos de Histogramas, Q-QPlots, Scatterplots e Boxplots (Maroco, 2007; Pallant, 2001). Os valores calculados confirmaram que a amostra em estudo cumpria os critérios de normalidade procedendo-se, neste sentido, a análises estatísticas mediante testes paramétricos. Na sequência dos objetivos propostos foram levadas a cabo análises de acordo valores de significância de $p<$ 0,05 para a interpretação dos dados. Destaca-se o recurso a análises correlacionais de Pearson, análises descritivas de médias e desvio padrão das variáveis em estudo, a análises de covariância multivariada (Mancova) para testagem de efeitos moderadores, e por último, pretendeu-se testar a presença de um efeito preditor entre as variáveis em estudo, recorrendo-se ao Modelo de Regressões Múltiplas Hierárquicas.

\section{Resultados}

\section{Associação entre o Coping e a Individuação aos Pais}

No que se refere às associações entre as dimensões do coping e a individuação à mãe, as análises destacam que o Coping Ativo e o Uso de Suporte Social e Emocional se correlacionam de forma positiva, embora fraca, com a Individuação Bem Sucedida e negativamente com a Negação das Necessidades de Vinculação. Por outro lado, o estilo de coping Negação apresenta correlação positiva moderada com a Negação das Necessidades de Vinculação e uma correlação negativa com a Individuação Bem Sucedida (Tabela 1).

No que diz respeito às associações entre as dimensões do coping e a individuação ao pai, destaca-se igualmente que o Coping Ativo e o Uso de Suporte Social e Emocional se correlacionam de forma positiva com a Manutenção do Laço e com a Individuação Bem Sucedida, denotando também uma correlação negativa, embora baixa, com a Negação das Necessidades de Vinculação. A dimensão de coping Negação revela associação significativa positiva moderada com a Negação das Necessidades de Vinculação e uma correlação negativa fraca com a Individuação Bem Sucedida (Tabela 1).

Tabela 1

Correlações de Pearson entre as Dimensões do Coping e a Individuação à Mãe e ao Pai

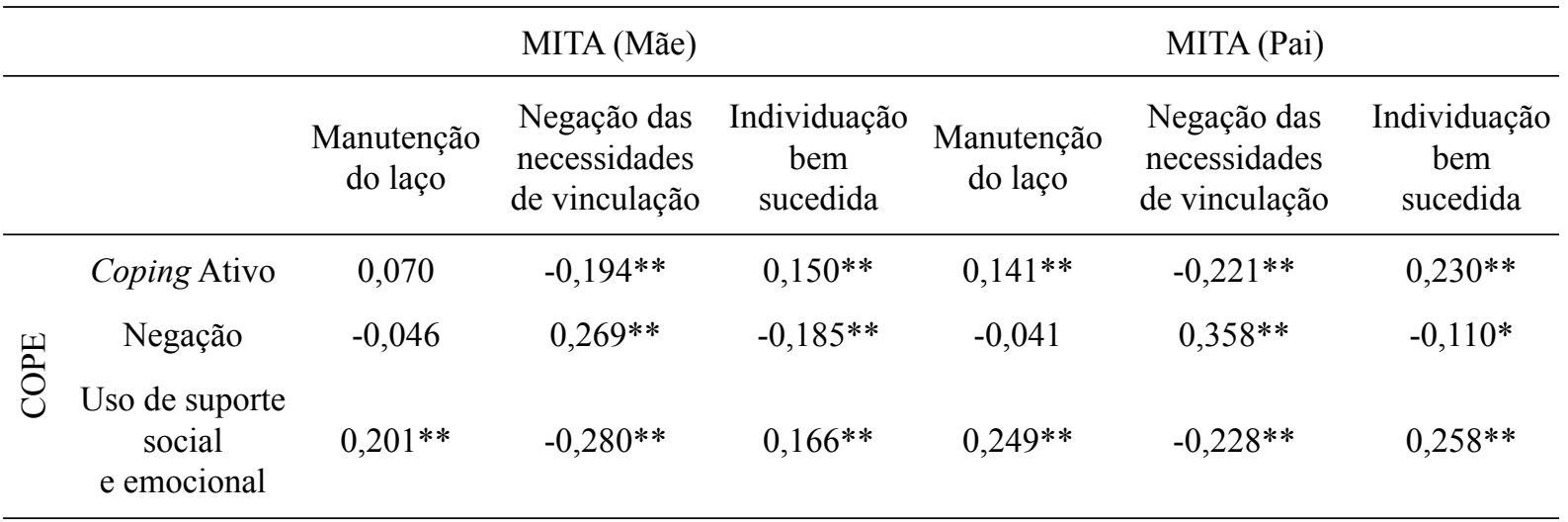

$* p<0,05 ; * * p<0,01$ 


\section{Efeito Preditor da Individuação aos Pais no Coping}

Procedeu-se ainda à realização de análises de regressão múltipla hierárquica com o intuito de analisar o contributo da individuação no coping. Neste sentido, foram introduzidos vários blocos adicionando à análise o efeito preditor das variáveis: gênero, configuração familiar, individuação à mãe e ao pai que foram sendo controladas na análise de regressão. No caso das variáveis gênero e configuração familiar procedeu-se à criação de variáveis dummy para analisar efeitos das categorias (sendo as codificações zero para o gênero masculino e um para o gênero feminino; zero para famílias divorciadas/separadas e um para famílias tradicionais).

$\mathrm{Na}$ análise de predição da dimensão do Coping Activo, duas variáveis apresentam contribuição significativa, nomeadamente a Individuação Bem Sucedida $(\beta=0,241)$ e a Negação das Necessidades de Vinculação $(\beta=-0,193)$ ao pai (Tabela 2). $\mathrm{Na}$ análise para a dimensão de coping Negação, apenas a variável Negação das Necessidades de Vinculação ao pai $(\beta=0,353)$ apresenta um contributo significativo (Tabela 2). Por último, a análise para a dimensão do coping através do Uso de Suporte Social e Emocional, sugere a predição através do contributo significativo das variáveis: género feminino $(\beta=0,165) \mathrm{e}$ Negação das Necessidades de Vinculação à mãe $(\beta=-0,196$; Tabela 2).

\section{Efeito Moderador da Configuração Fa- miliar na Associação entre a Individua- ção aos Pais e o Coping}

De encontro aos objetivos do estudo, foram ainda realizadas análises de covariância multivariada (MANCOVAS) para verificar o efeito moderador da configuração familiar na associação entre a individuação e o coping. Para a realização desta análise, procedeu-se ao cálculo da média das dimensões da individuação à mãe e ao pai (MITA) e do coping (COPE), com o intuito de poder categorizar cada uma das dimensões em alto e baixo nível. No que se refere à individuação à mãe obteve-se uma média das dimensões Relatedness de $M=2,9$, enquanto que para o pai a média foi de $M=2,7$. Assim, os par- ticipantes que apresentam um elevado nível de individuação bem sucedida revelam uma média acima desses valores, enquanto os participantes que têm um baixo nível de individuação revelam média inferior ou igual aos respectivos valores. No COPE obteve-se uma média de Adaptação Social de $M=3,9$, sendo que os participantes que apresentarem média superior a $M=3,9$ desenvolvem estratégias mais adaptadas de coping, encontrando-se melhor adaptados socialmente, enquanto que aqueles que apresentem média menor ou igual a 3,9 revelam maiores dificuldades de adaptação psicossocial. Nesta análise verificou-se a inexistência de um efeito moderador da Configuração Familiar, $F_{\text {mãe }}(5,294)=0,406 ; p=$ 0,$845 ; F_{\text {pai }}(5,363)=0,759 ; p=0,580$, para mãe e pai respectivamente. Portanto verificou-se que a individuação é melhor preditora do coping independentemente da configuração familiar nesta associação.

\section{Discussão}

De acordo com os objetivos do presente estudo analisou-se a existência de associações entre a individuação e o coping, assim como o contributo da individuação no desenvolvimento do coping, sendo testado ainda o efeito moderador da configuração familiar na associação anterior.

Neste sentido, verificou-se a existência de associações entre a individuação à mãe e ao pai com as dimensões relativas ao coping. No que se refere à dimensão Manutenção do Laço tanto na versão mãe como para o pai encontra-se associada de forma positiva ao Uso de Suporte Social e Emocional. Sendo que na versão pai a Manutenção do Laço encontra-se também associada de forma positiva com o Coping Ativo. A manutenção do laço diz respeito ao desejo de proximidade física e emocional, bem como procura de apoio (Kruse \& Walper, 2008), indo de encontro à estratégia de coping que apela à necessidade de obter apoio moral. Assim, a existência de ligações significativas e estáveis com os pais leva o adolescente a se sentir mais seguro (Amato \& Sobolewski, 2001) e que se sinta confiante para procurar esse apoio. De acordo com Ainsworth (1969) e Bowlby (1988), a proximidade física e 
Tabela 2

Análise de Regressão Múltipla Hierárquica para o Coping

\begin{tabular}{|c|c|c|c|c|c|c|}
\hline & $R^{2}$ & $R^{2}$ Change & $\mathrm{B}$ & SE & $\beta$ & $p$ \\
\hline \multicolumn{7}{|l|}{ COPING ACTIVO } \\
\hline $\begin{array}{l}\text { Bloco } 1 \\
\text { Gênero (dummy) }\end{array}$ & 0,007 & 0,007 & & & & \\
\hline $\begin{array}{l}\text { Bloco } 2 \\
\quad \text { Configuração Familiar (dummy) }\end{array}$ & 0,011 & 0,004 & & & & \\
\hline Bloco 3 - MITA (mãe) & 0,054 & 0,043 & & & & \\
\hline \multicolumn{7}{|l|}{ Individuação Bem Sucedida } \\
\hline \multicolumn{7}{|l|}{ Neg. Necessidades de Vinculação } \\
\hline \multicolumn{7}{|l|}{ Manutenção do Laço } \\
\hline Bloco 4 - MITA (pai) & 0,083 & 0,029 & & & & \\
\hline Individuação Bem Sucedida & & & 0,297 & 0,119 & 0,241 & 0,013 \\
\hline Neg. Necessidades de Vinculação & & & $-0,281$ & 0,128 & $-0,193$ & 0,029 \\
\hline \multicolumn{7}{|l|}{ Manutenção do Laço } \\
\hline \multicolumn{7}{|l|}{ NEGAÇÃO } \\
\hline $\begin{array}{l}\text { Bloco } 1 \\
\text { Gênero (dummy) }\end{array}$ & 0,008 & 0,008 & & & & \\
\hline $\begin{array}{l}\text { Bloco } 2 \\
\quad \text { Configuração Familiar (dummy) }\end{array}$ & 0,008 & 0,000 & & & & \\
\hline Bloco 3 - MITA (mãe) & 0,095 & 0,087 & & & & \\
\hline \multicolumn{7}{|l|}{ Individuação Bem Sucedida } \\
\hline \multicolumn{7}{|l|}{ Neg. Necessidades de Vinculação } \\
\hline \multicolumn{7}{|l|}{ Manutenção do Laço } \\
\hline Bloco 4 - MITA (pai) & 0,138 & 0,042 & & & & \\
\hline \multicolumn{7}{|l|}{ Individuação Bem Sucedida } \\
\hline Neg. Necessidades de Vinculação & & & 0,633 & 0,153 & 0,353 & 0,001 \\
\hline \multicolumn{7}{|l|}{ Manutenção do Laço } \\
\hline \multicolumn{7}{|l|}{ SUPORTE SOCIAL E EMOCIONAL } \\
\hline $\begin{array}{l}\text { Bloco } 1 \\
\quad \text { Gênero (dummy) }\end{array}$ & 0,039 & 0,039 & 0,402 & 0,120 & 0,165 & 0,001 \\
\hline $\begin{array}{l}\text { Bloco } 2 \\
\quad \text { Configuração Familiar (dummy) }\end{array}$ & 0,040 & 0,001 & & & & \\
\hline Bloco 3 - MITA (mãe) & 0,126 & 0,086 & & & & \\
\hline \multicolumn{7}{|l|}{ Individuação Bem Sucedida } \\
\hline Neg. Necessidades de Vinculação & & & $-0,372$ & 0,164 & $-0,196$ & 0,024 \\
\hline \multicolumn{7}{|l|}{ Manutenção do Laço } \\
\hline Bloco 4 - MITA (pai) & 0,142 & 0,016 & & & & \\
\hline \multicolumn{7}{|l|}{ Individuação Bem Sucedida } \\
\hline \multicolumn{7}{|l|}{ Neg. Necessidades de Vinculação } \\
\hline Manutenção do Laço & & & & & & \\
\hline
\end{tabular}


emocional criada entre a figura cuidadora poderá facultar sentimentos de segurança traduzindo-se também na consciencialização de que perante as angústias sentidas poderá socorrer-se dessa figura significativa, permitindo-lhe uma exploração do meio menos inibida, desenvolvendo a sua autonomia. Assim, experiências de qualidade num contexto familiar favorável proporciona uma individuação bem sucedida, sendo que a segurança desenvolvida no seio da relação materna pode estar associada ao desenvolvimento de estratégias de coping mais adaptativas e funcionais. Buhl (2008b) enfatiza esta ideia de que a qualidade das ligações com as figuras parentais conduz a sentimentos de afeição e estabilidade que levam ao processo de individuação. A relação inicial estabelecida com os cuidadores primários parece, assim, ligar-se fortemente com o desenvolvimento emocional e relacional dos adolescentes, fornecendo-lhe recursos para fazer face aos eventos geradores de stress.

A dimensão Individuação Bem Sucedida refere-se à capacidade do indivíduo manter o equilíbrio nas suas relações significativas em termos de autonomia e proximidade (Kruse \& Walper, 2008). Tanto na versão mãe como para o pai, esta dimensão surge associada de forma positiva com todas as dimensões positivas do coping (Coping Ativo e Uso de Suporte Social e Emocional) e de forma negativa com a dimensão negativa do coping (Negação). A individuação bem sucedida apresenta-se estreitamente relacionada com a qualidade da vinculação estabelecida com as figuras significativas de afeto. Assim, na contingência de um evento stressor essas figuras poderão constituir uma fonte de apoio moral, compreensão, verificando-se nesta medida uma associação entre todas as dimensões, assim como, dependendo da relação estabelecida com essas figuras significativas poderá desenvolver ou não estratégias de coping mais adaptativas e funcionais. A partir da perceção que os adolescentes têm das suas figuras parentais, como sendo modelos de vinculação disponíveis e responsivos vão criando uma imagem de si como sendo figuras queridas e amadas conduzindo a um processo de separação-individuação mais bem sucedido. Por outra parte, se os jovens não se sentem seguros poderão desenvolver estratégias menos adaptativas como evitar encarar ou rejeitar os acontecimentos. A existência de interações negativas e indisponibilidade parental pode conduzir à não perceção das necessidades emocionais dos adolescentes, podendo recriar nos mesmos uma insegurança interna (Harold et al., 2013). De acordo com Marcelli e Braconnier (2005) o processo de individuação parece modificar o equilíbrio entre a vinculação e a autonomia, o qual se encontra associado ao sentimento de segurança desenvolvido pelos adolescentes e da forma como se encontram preparados para a tarefa de individuação. Os laços emocionais criados precocemente irão assim facultar ao adolescente maior confiança e segurança permitindo-lhe uma individuação bem sucedida (van Wel, ter Bogt, \& Raaijmakers, 2001), facilitando o desenvolvimento de melhores estratégias na resolução de situações geradoras de stress (Arndt \& Goldenberg, 2002). É durante a adolescência que se desenvolvem habilidades de coping (construídas a partir de experiências precoces) que serão aplicadas na interpretação e gestão das situações de stress no futuro (Seiffge-Krenke \& Beyers, 2005). Assim, os adolescentes adotam estratégias de coping mais adaptativas consoante as representações mentais criadas com base nas experiências precoces de vinculação. A capacidade dos adolescentes para lidarem com o processo de divórcio pode depender da relação estabelecida dos pais enquanto cônjuges e pais (Mota \& Matos, 2013). Assim, a forma como os adolescentes encaram o divórcio está associado à forma como os pais gerem os conflitos na sua própria relação conjugal e com a disponibilidade pessoal que assumem na relação como filhos (Hack \& Ramires, 2010).

Ainda nos resultados observados, a Negação das Necessidades de Vinculação traduz certa retratividade na procura de apoio por parte dos adolescentes (Kruse \& Walper, 2008), sendo que no presente estudo, a versão mãe e pai se encontram associados de forma negativa com as dimensões positivas do coping (Coping Ativo e Uso de Suporte Social e Emocional); enquanto que com a dimensão Negação do coping apresenta uma associação positiva. Desta forma, su- 
gere-se que os adolescentes podem desenvolver processos defensivos evitando aceitar a necessidade de proximidade e suporte emocional, o que pode colocar um entrave ao desenvolvimento de sentimentos de segurança e confiança que lhes permita utilizar estratégias de coping adaptativas e funcionais. Neste sentido, os adolescentes parecem desenvolver menos estratégias de $\mathrm{CO}^{-}$ ping positivas, como a procura de informação, mobilização de recursos sociais, apoio moral, compreensão, que o poderiam auxiliar face às adversidades, prevalecendo mais estratégias negativas. Assim, está implícita uma dificuldade pessoal aquando de situações ansiogénicas. $\mathrm{O}$ desenvolvimento de sentimentos de defesa poderão estar ligados a relações precoces inseguras, dificultando a aprendizagem em lidar com as suas angústias. Howard e Medway (2004) defendem que adolescentes inseguros parecem revelar estratégias de coping de evitamento perante conflitos. Mota e Matos (2010) constataram que adolescentes menos defensivos parecem apresentar maior disponibilidade emocional, o que se reflete na qualidade das relações estabelecidas com os outros e no desenvolvimento de competências sociais que lhes permitem a procura de apoio.

O presente estudo observa ainda resultados inerentes ao contributo da individuação parental no desenvolvimento do coping. $\mathrm{Na}$ dimensão Coping Activo verificaram-se contributos significativos da Individuação Bem Sucedida ao pai de forma positiva e Negação das Necessidades de Ligação ao pai de forma negativa. Os resultados sugerem, assim, que os adolescentes que apresentam melhores índices de segurança, e portanto desenvolvem uma individuação bem sucedida, adquirem estratégias de coping adaptativas como é o caso do coping ativo, procurando auxílio e apoio perante as dificuldades. Já aqueles que apresentam sentimentos de retração face ao pai parecem assumir significativamente estratégias de coping de negação. De acordo com a literatura, os adolescentes que percebem a família como sendo um suporte desenvolvem estratégias de coping ativas, ao invés, o sentimento de ausência emocional poderá levar à adoção de uma postura de "defesa", condicionando a elaboração de estratégias de coping adaptativas (e.g., Cabral
\& Matos, 2007; Seiffge-Krenke, 2011; Seiffge-Krenke \& Beyers, 2005). Assor e Tal (2012) tinham já destacado a influência da qualidade da vinculação na manifestação de comportamentos retrativos e dificuldades na gestão do stress na adolescência. A qualidade das relações estabelecidas precocemente e que levam ao desenvolvimento de uma vinculação segura irão, por sua vez, permitir uma individuação bem sucedida que possibilitará ao adolescente desenvolver relações positivas com o exterior.

Para a dimensão Negação do coping verifica-se a contribuição significativa positiva da Negação das Necessidades de Vinculação ao pai. Assim, os adolescentes que desenvolvem estratégias de coping defensivas e mal adaptativas, evitando o envolvimento e enfrentamento de situações geradoras de stress parecem ter desenvolvido relações de vinculação insegura (DiFilippo \& Overholser, 2000). A criação de modelos internos negativos conduz uma vinculação ansiosa/insegura, revelando nos indivíduos comportamentos evitantes ou resistentes perante a preocupação sentida com a indisponibilidade e não responsivadade das figuras de vinculação (e.g. Mota \& Matos, 2013). Komidar e colaboradores (2013), sublinham que o desenvolvimento de relações inseguras poderá manifestar-se na relação com os outros, levando a dificuldades no processo de autonomia. No período da adolescência é frequente que o adolescente vivencie sentimentos de ambivalência, num misto de desejo de separação e proximidade com as figuras cuidadoras (Fleming, 2005; Smith, Calkins, Keane, Anastopoulos, \& Shelton, 2004). Assim, é também importante que o adolescente se sinta ligado aos pais num sistema vinculativo saudável no sentido de atravessar um processo de separação-individuação bem sucedida (Fleming, 2005; Komidar et al., 2013). Por outro lado, a adoção de um comportamento de isolamento e centrado em si poderá constituir-se como uma estratégia de adaptação menos positiva perante a situação de ausência física e/ou emocional da figura parental (Holmes, 2001).

No Uso de Suporte Social e Emocional, a variável gênero feminino apresenta um contributo significativo. Alguns autores têm vindo a 
corroborar este dado ao constatarem que existem diferenças nos processos de coping consoante o gênero, sendo que adolescentes do género feminino recorrem mais frequentemente à busca de apoio social, moral, compreensão e acções diretas (Seiffge-Krenke, 2011; Seiffge-Krenke \& Shulman, 1990). Segundo a literatura, existem diferenças entre gêneros, sublinhando-se que o gênero feminino passa por um processo de socialização diferente do gênero masculino (Lisboa et al., 2002). De acordo com Zimmermann e Iwanski (2014), o gênero feminino parecem ter maior facilidade em procurar suporte social, aceitando ajuda externa, o que se repercute num processo de autonomia ao longo da adultícia. Os autores apontam ainda que as adolescentes do gênero feminino parecem adotar estratégias de coping mais focadas na emoção em comparação com os rapazes, estando geralmente mais disponíveis para procurar solucionar os problemas de forma verbal, assim como para expressar sentimentos e ideias (Mandl, 2007). A variável Negação das Necessidades de Vinculação à mãe apresenta também uma contribuição significativa negativa no Uso de Suporte Social e Emocional, sendo que o adolescente ao negar a existência das suas necessidades de proximidade, poderá desenvolver comportamentos de evitamento e evitar a procura de apoio (Seiffge-Krenke \& Beyers, 2005). Os mesmos autores sublinham que os adolescentes que sentem a sua família como um suporte estão mais disponíveis para utilizar estratégias de coping ativas.

Os resultados deste estudo demonstraram ainda que a configuração familiar não revela um papel moderador na associação entre a individuação aos pais e o coping. Tal como seria esperado a individuação prediz melhor o desenvolvimento de estratégias de coping independentemente da configuração familiar. Este resultado não deixa de ser interessante na medida em que destaca a importância do investimento e disponibilidade das figuras parentais, ao invés da sua condição conjugal. A qualidade da vinculação aos pais parece conduzir a uma perceção de segurança emocional que promove a capacidade adaptativa do adolescente. Neste seguimento, Hack e Ramires (2010) sublinham que a manutenção das figuras parentais numa relação conjunta não promove necessariamente a saúde mental dos adolescentes, na medida em que relações pautadas pelos conflitos interparentais sugerem menor disponibilidade e responsividade na relação com os filhos (Amato \& Afifi, 2006; Benetti, 2006; Mota $\&$ Matos, 2013).

Desta forma, os resultados apontam para a importância da qualidade da vinculação desenvolvida com as figuras parentais, uma vez que são estes laços que irão criar um suporte emocional capaz de facilitar o desenvolvimento do coping. Esta questão tem vindo a ser largamente suportada pela literatura (e.g. Holmes, 2001; Mota \& Matos, 2009; Seiffge-Krenke \& Beyers, 2005), na medida em que a qualidade da vinculação às figuras cuidadoras facilita o processo de separação-individuação (Arnett, 2007). Para Allen e colaboradores (2003) os adolescentes seguros apresentam maior disponibilidade para a exploração da sua autonomia, pois percebem que os pais irão manter a relação independentemente das descontinuidades. Assim, a configuração familiar vem perdendo relevância em detrimento da relação estabelecida precocemente com os pais. Os efeitos do divórcio dependem mais da maneira que o casal gere todo o processo (Mathew et al., 2011; Orbuch, Thornton, \& Cancio, 2000). Por vezes a separação parental leva à conquista de menores conflitos o que pode ser mais benéfico para os filhos em termos de segurança e autonomia comparativamente aos filhos que vivem em famílias pautadas por conflitos parentais (Amato, 2010; Moura \& Matos, 2008).

\section{Limitações e Investigações Futuras}

O presente estudo pretendeu clarificar que o processo de divórcio enquanto transição familiar por si só não apresenta condicionantes lineares para o processo de individuação dos adolescentes, podendo existir outros fatores que interferem nesse processo, facultando resultados que poderão servir como ponto de partida para futuras investigações de aprofundamento na área. Em futuras investigações seria pertinente incluir novas variáveis, nomeadamente a qualidade da vinculação aos irmãos e pares amigos e a sua associação com os processos de separação-indivi- 
duação. Seria ainda de todo relevante incluir dimensões ligadas à dinâmica familiar como sendo os conflitos interparentais. Poderia sugerir-se a compreensão da replicação desses padrões em relações posteriores, nomeadamente com o par amoroso. Algumas limitações do estudo prenderam-se com a dimensão da amostra, pelo que seria importante que houvesse uma distribuição o mais igualitária possível dos participantes que provêm de famílias tradicionais e divorciadas, concretizando um estudo mais representativo do país, englobando adolescentes de diferentes locais geográficos; assim como uma melhor homogeneidade em termos de gênero. Outra limitação relevante relaciona-se com o recurso a questionários de auto-relato que poderá ter inerente o enviesamento dos resultados, associados a fatores externos, como fadiga e desejabilidade social. Por último, o fato de se tratar de um estudo transversal não permitiu perceber a relação de causalidade das variáveis estudadas sendo por isso relevante em estudos futuros desenvolver uma análise longitudinal.

\section{Referências-}

Ainsworth, M. D. S. (1969). Object relations, dependency, and attachment: A theoretical review of the infant-mother relationship. Child Development, 40, 969-1025.

Allen, J. P., MacElhaney, K. B., Land, D. J., Kupermic, G. P., Moore, C. W., O’Beeirne, H., \& Kilmer, S. L. (2003). A secure base in adolescence: Markers of attachment security in the mother-adolescent relationship. Child Development, 74, 292-307. doi:10.1111/1467-8624.t01-1-00536

Amato, P. R. (2010). Research on divorce: Continuing trends and new developments. Journal of Marriage and the Family, 72, 650-666. doi:10.1111/j.1741-3737.2010.00723.x

Amato, P. R., \& Afifi, T. D. (2006). Feeling caught between parents: Adult children's relations with parents and subjective well-being. Journal of Marriage and Family, 68(1), 222-235. doi:10.1111/j.1741-3737.2006.00243.x

Amato, P. R., \& Cheadle, J. (2008). Parental divorce, marital conflict and children's behavior problems: A comparison of adopted and biological children. Social Forces, 86(3), 1139-1161. doi:10.1353/sof.0.0025
Amato, P. R., \& Sobolewski, J. M. (2001). The effects of divorce and marital discord on adult children's psychological well-being. American Sociological Review, 66(6), 900-921.

Arndt, J., \& Goldenberg, J. L. (2002). From threat to sweat: The role of psysiological arousal in the motivation to maintain self-esteem. In A. Tesser, D. A. Stapel, \& J. V. Wood (Eds.), Self and motivation: Emerging psychological perspectives (pp. 43-69). Washington, DC: American Psychological Association.

Arnett, J. J. (2007). Emerging adulthooh: What is it and what is it good for? Journal Compilation, 1(2), 68-73. doi:10.1111/j.1750-8606.2007.00016.x

Assor, A., \& Tal, K. (2012). When parents' affection depends on child's achievement: Parental conditional positive regard, self-aggrandizement, shame and coping in adolescents. Journal of Adolescence, 35, 249-260. doi:10.1016/j.adolescence.2011.10.004

Benetti, S. P. C. (2006). Conflito conjugal: Impacto no desenvolvimento psicológico da criança e do adolescente. Psicologia: Reflexão e Crítica, 19, 261-268.

Bowlby, J. (1988). A secure base: Parent-child attachment and healthy human development. London: Basic Books.

Buhl, H. M. (2008a). Significance of individuation in adult child-parent relationships. Journal of Family Issues, 29, 262-281. doi:10.1177/ 0192513X07304272

Buhl, H. M. (2008b). Development of a model describing individuated adult child-parent relationships. International Journal of Behavioural Development, 32, 381-389. doi:10.1177/ 0165025408093656

Cabral, J., \& Matos, P. (2004). COPE. Adaptação para a população portuguesa. Manuscrito não publicado, Faculdade de Psicologia e de Ciências da Educação, Universidade do Porto, Portugal.

Cabral, J., \& Matos, P. M. (2007). Attachment and adjustment to college: The mediating role of emotion regulation and coping. Paper presented at the $10^{\text {th }}$ European Congress of Psychology, Praga, Chec Republic.

Cano, D., Gabarra, L., Moré, C., \& Crepaldi, M. (2009). As transições familiares do divórcio ao recasamento no contexto brasileiro. Psicologia: Reflexão e Crítica, 22(2), 214-222. 
Carver, C. S., Scheier, M. F., \& Kumari Weintraub, J. (1989). Assessing coping strategies: A theoretically based approach. Journal of Personality and Social Psychology, 56(2), 267-283.

Coburn, K. L. (2006). Organizing a ground crew for today's helicopter parents. About Campus, 11, 9-16. doi:10.1002/abc.167

Cohen, P., Kasen, S., Chen, H., Hartmark, C., \& Gordon, K. (2003). Variations in patterns of developmental transitions in the emerging adulthood period. Developmental Psychology, 39, $657-$ 669. doi:10.1037/0012-1649.39.4.657

Costa, M., \& Mota, C. P. (2012). Configuração familiar, género e coping em adolescentes: Papel dos pares. Psicologia em Estudo, 17(4), 567-575. doi:10.1590/S1413-73722012000400003

DiFilippo, J. M., \& Overholser, J. C. (2000). Suicidal ideation in adolescent psychiatric inpatients as associated with depression and attachment relationships. Journal of Clinical Child Psychology, 29, 155-166. doi:10.1207/S15374424jccp2902_2

Eccles, J., Templeton, J., Barber, B., \& Stone, M. (2003). Adolescence and emerging adulthood: The critical passage ways to adulthood. In M. H. Bornstein, L. Davidson, C. L. M. Keyes, \& K. A. Moore (Eds.), Well-beings: Positive development across the life course. Mahwah, $\mathrm{NJ}$ : Lawrence Erlbaum.

Fleming, M. (2005). Entre o medo e o desejo de crescer: Psicologia da adolescência. Porto, Portugal: Afrontamento.

Folkman, S., \& Moskowitz, J. T. (2004). Coping: Pitfalls and promise. Annual Review Psychological, 55, 745-774. doi:10.1146/annurev. psych.55.090902.141456

Hack, S., \& Ramires, V. (2010). Adolescência e divórcio parental: Continuidades e rupturas dos relacionamentos. Psicologia Clinica, 22(1), 8597. doi:10.1590/S0103-56652010000100006

Harold, G., Leve, L., Elam, K., Thapar, A., Neiderhiser, J., Ntsuaki, M., ...Reiss, D. (2013). The nature of nurture: Disentangling passive genotype-environment correlation from family relationship influences on children's externalizing problems. Journal of Family Psychology, 27(1), 12-21. doi:10.1037/a0031190

Holmes, J. (2001). The search for the secure base. Attachment theory and psychoterapy. Philadelphia, PA: Taylor \& Francis.
Howard, M. S., \& Medway, F. J. (2004). Adolescents' attachment and coping with stress. Psychology in the Schools, 41, 391-402. doi:10.1002/ pits. 10167

Komidar, L., Zupančič, M., Sočan, G., \& Puklek Levpušček, M. (2013). Development and construct validation of the Individuation Test for Emerging Adults (IT-EA). Journal of Personality Assessment, 1-12. doi:10.1080/00223891.20 13.850703

Kruse, J., \& Walper, S. (2008). Types of individuation in relation to parents: Predictors and outcomes. International Journal of Behavioral Development, 32(5), 390-400. doi:10.1177/0165025408093657

Lansu, T. A., \& Cillessen, A. H. (2012). Peer status in emerging adulthood: Associations of popularity and preference with social roles and behavior. Journal of Adolescent Research, 27(1), 132-150. doi:10.1177/0743558411402341

Larkin, K. T., Frazer, N. L., \& Wheat, A. L. (2011). Responses to interpersonal conflict among young adults: Influence of family of origin. Personal Relationships, 18, 657-667. doi:10.1111/ j.1475-6811.2010.01334.x

Lisboa, C., Koller, S. H., Ribas, F. F., Bitencourt, K., Oliveira, L., Porciuncula, L. P., \& Marchi, R. B. (2002). Estratégias de coping de crianças vítimas e não vítimas de violência doméstica. Psicologia: Reflexão e Crítica, 15(2), 345-362.

Mandl, M. C. (2007). The relationship between adolescente parental attachment, curiosity, and coping with stress (Unpublished doctoral thesis). EDT Collection for Wayne State University, Detroit, MI.

Marcelli, D., \& Braconnier, A. (2005). Adolescência e psicopatologia. Lisboa: Climepsi.

Maroco, J. (2007). Análise estatística com utilização do SPSS. Lisboa: Sílabo.

Mathew, D., Gayman, M. D., Turner, R. J., Cislo, A. M., \& Eliassen, A. H. (2011). Early adolescent family experiences and perceived social support in young adulthood. Journal of Early Adolescence 31(6), 880-908. doi:10.1177/0272431610376247

Saraiva, L., Brandão, T., \& Matos, P. M. (2010). Factor structure of the Portuguese version of MITA. Comunicação apresentada no Paper presented at the 5th Congress of the European Society on Family Relations. Milão, Itália. Retrie- 
ved from http://www.yagiss.de/en/downloads/ ESFR_2010_Saraiva_Matos.pdf

Mayseless, O., \& Scharf, M. (2009). Too close for confort: Inadequate boundaries with parents and individuation in late adolescent girls. American Journal of Orthopsychiatry, 79, 191-202. doi:10.1037/a0015623

Melo, O., \& Mota, C. P. (2014). Interparental conflicts and psychopatological development in adolescents and young adults. Paidéia (Ribeirão Preto), 24(59), 283-293. doi:10.1590/198243272459201402

Mota, C. P., \& Matos, P. M. (2009). Apego, conflito e auto-estima em adolescentes de famílias tradicionais e divorciadas. Psicologia: Reflexão $e$ Crítica, 22(3), 317-325.

Mota, C. P., \& Matos, P. M. (2010). Adolescentes institucionalizados: O papel das figuras significativas na predição da assertividade, empatia e autocontrolo. Análise Psicológica, 2(28), $245-$ 254. doi:10.14417/ap.278

Mota, C. P., \& Matos, P. M. (2013). Conflitos interparentais e processos de individuação em adultos emergentes portugueses: $\mathrm{O}$ papel dos conflitos de lealdade. Psicologia: Teoria $e$ Pesquisa, 29(3), 267-276. doi:10.1590/S010237722013000300004

Mota, C. P., \& Rocha, M. (2012). Crescimento pessoal na adolescência e jovem adultícia: Separação-individuação e o jogo das relações. Psicologia: Teoria e Pesquisa, 28(3), 357-367. doi:10.1590/ S0102-37722012000300011

Moura, O., \& Matos, P. M. (2008). Vinculação aos pais, divórcio e conflito inter-parental em adolescentes. Psicologia, 22(1), 127-152.

Orbuch, T. L., Thornton, A., \& Cancio, J. (2000). The impact of marital quality, divorce, and remarriage on the relationships between parents and their children.Marriage \& FamilyReview, 29, 221-246.

Pallant, J. (2001). SPSS survival manual. Buckingham, UK: Open University Press.

Ramires, V. (2004). As transições familiares: A perspectiva de crianças e pré-adolescentes. Psicologia em Estudo, 9(2), 183-193. doi:10.1590/ S1413-73722004000200005

Rocha, M., \& Matos, P. M. (2012). Componentes de apego em adolescentes portugueses. Revista Argentina de Clínica Psicológica, 21, 197-208.

Scabini, E., \& Manzi, C. (2011). Family processes and identity. In S. J. Schwartz, K. Luyckx, \& V.
L. Vignoles (Eds.), Handbook of identity theory and research (Vol. 2, pp. 565-584). New York: Springer.

Seiffge-Krenke, I. (2011). Coping with relationship stressors: A decade review. Journal of Research on Adolescence, 21(1), 196-210. doi:10.1111/ j.1532-7795.2010.00723.x

Seiffge-Krenke, I., \& Beyers, W. (2005). Coping trajectories from adolescence to young adulthood: Links to attachment state of mind. Jounal of Research on Adolescence, 15(4), 561-582. doi:10.1111/j.1532-7795.2005.00111.x

Seiffge-Krenke, I., \& Shulman, S. (1990). Coping style in adolescence: A cross-cultural study. Journal of Cross-Cultural Psychology, 21, 351-377.

Smith, C. L., Calkins, S. D., Keane, S. P., Anastopoulos, A. D., \& Shelton, T. L. (2004). Predicting stability and change in toddler behavior problems: Contributions of maternal behavior and child gender. Developmental Psychology, 40(1), 29-42.

Van Petegem, S., Beyers, W., Brenning, K., \& Vansteenkiste, M. (2013). Exploring the association between insecure attachment styles and adolescent autonomy in family decision making: A differentiated approach. Journal of Youth \& Adolescence, 42(12), 1837-1846. doi:10.1007/ s10964-012-9886-0

Van Wel, F., ter Bogt, T., \& Raaijmakers, Q. (2001). Changes in the parental bond and the well-being of adolescentes and young adults. Adolescence, $37(146), 317-333$.

Walper, S., Schwarz, B., \& Jurasic, S. (1996). Entwicklung und Erprobung des Münchner Individuationstests (Berichte aus der Arbeitsgruppe, Familienentwicklung nach der Trennung' 8/1996) [Munich Inventory Test of Adolescence - MITA]. Unveröffentlichtes Manuskript, Ludwig-Maximilians-Universität München, Deutschland.

Zimmermann, P., \& Iwanski, A. (2014). Emotion regulation from early adolescence to emerging adulthood and middle adulthood: Age differences, gender differences, and emotion-specific developmental variations. International Journal of Behavioral Development, 38, 182-194. doi:10.1177/0165025413515405

Recebido: 04/05/2015

$1^{a}$ revisão: 28/05/2015

$2^{a}$ revisão: 1\%09/2015

Aceite final: 24/09/2015 